\title{
MEASUREMENTS OF OUTBURST CHARACTERISTICS, TEMPERATURES, DENSITIES AND ABUNDANCES IN THE EJECTA OF NOVA MUSCAE 1983.
}

\author{
B.J.M. Hassall, RGO, Cambridge \\ M.A.J. Snijders, Astronomisches Institut, Tubingen \\ A. W. Harris, RAL, Chilton \\ A. Cassatella, ESA IUE Observatory, Madrid \\ M. Dennefeld, M. Friedjung, Paris \\ M. Bode, D. Whittet, Lancaster Polytechnic \\ P. Whitelock, J. Menzies, T. Lloyd Evans, SAAO, Cape Town \\ G.T. Bath, Dept of Astrophysics, Oxford
}

Introduction. We present results for IUE, optical and IR observations of Nova Muscae 1983, from early outburst to January 1986 obtained by the European IUE Target of Opportunity Team. A detailed description of the data will appear elsewhere (Hassall et al., 1989), but here we summarise the most important results.

The outburst lightcurve initially indicated a fast speed class for this nova, but was later characterised by a rather slow optical decline with two or more secondary outbursts with sudden doubling of the bolometric flux. In Figure 1, we show the contributions of X-ray, UV, optical and IR to the total luminosity for 1200 days following outburst, assuming a distance of $4.3 \mathrm{kpc}$ and an interstellar extinction $E(B-V)=0.5$. In the absence of dust formation, first the UV and later the $\mathrm{X}$-ray flux (Ögelman et al, 1984) dominate the radiative energy late into the nebular phase. There was a plateau stage lasting about 500 days, with a bolometric luminosity of $\sim 10^{38} \mathrm{ergs} \mathrm{s}^{-1}$ near the Eddington limit. The secondary outbursts were thus super-Eddington. We estimate a total outburst energy (including kinetic and gravitational potential energy of the ejecta) of $\sim 5 \times 10^{46}$ ergs, corresponding to a mass of $\sim 4 \times 10^{-6} M_{0}$ of hydrogen burnt in the thermonuclear runaway.

Figure 1:- Nova Muscae bolometric lightcurve

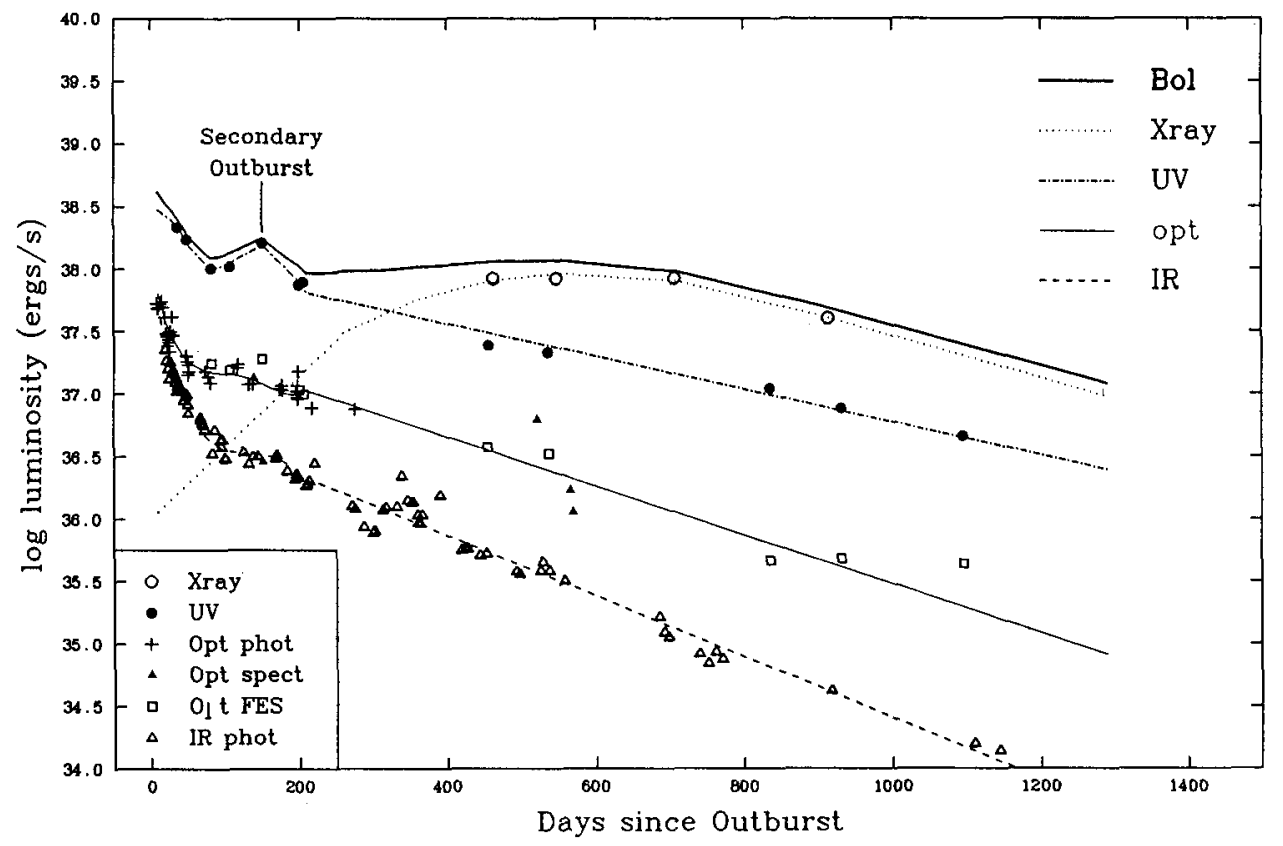


Electron Temperatures and Densities. Because of the large simultaneous wavelength coverage, many line ratios have been available at most stages of the nova decline, for both temperature and density determination. The electron temperature can be derived from the flux ratios of collisionally excited to dielectronic recombination lines (Stickland et al. 1981, Storey 1981). The temperatures derived from the $\mathrm{N} 4+$ recombination lines are generally higher than those emitting $\mathrm{C} 2+$ and $\mathrm{C} 3+,(\sim 13500 \mathrm{~K}$ and $\sim 11000 \mathrm{~K}$ respectively on Day 455$)$ indicating stratification in the ejected shell. The electron temperature showed a general increase with time.

Table 1; Abundances by Number

\begin{tabular}{|c|c|c|c|}
\hline & $\begin{array}{l}\text { Solar } \\
\text { System }\end{array}$ & $\begin{array}{l}\text { Nova } \\
\text { Mus } 83\end{array}$ & $\begin{array}{l}\text { Over } \\
\text { Abundance }\end{array}$ \\
\hline $\operatorname{Ref}$ & (1) & & \\
\hline $\mathrm{H}$ & 1.0 & 1.0 & 1.0 \\
\hline $\mathrm{He}$ & $6.8 \mathrm{E}-2$ & 2. $9 \mathrm{E}-1$ & 4.2 \\
\hline $\mathrm{C}$ & $4.2 \mathrm{E}-4$ & $4.9 E-3$ & 11.7 \\
\hline $\mathrm{N}$ & $8.7 E-5$ & $5.1 \mathrm{E}-2$ & 588.4 \\
\hline 0 & $6.9 E-4$ & $4.3 E-2$ & 62.4 \\
\hline $\mathrm{Ne}$ & $9.8 E-5$ & $6.3 E-4$ & 6.4 \\
\hline $\mathrm{Na}$ & $2.3 E-6$ & - & - \\
\hline $\mathrm{Mg}$ & $4.0 \mathrm{E}-5$ & $2.2 E-4$ & 5.4 \\
\hline Al & $3.2 \mathrm{E}-6$ & $<7.6 \mathrm{E}-5$ & $<23.9$ \\
\hline Si & $3.8 E-5$ & 3. $6 \mathrm{E}-4$ & 9.5 \\
\hline $\mathrm{s}$ & $1.9 E-5$ & $1.8 E-4$ & 9.6 \\
\hline $\mathrm{Fe}$ & $3.4 \mathrm{E}-5$ & $3.1 E-5$ & 0.9 \\
\hline
\end{tabular}

Reference: (1) Cameron (1982)
The NIII] $1750 \mathrm{~A}$ intercombination quintet is a useful density diagnostic and gives results about an order of magnitude higher than the optical [OI] and [OIII] density $\left(\sim 10^{7.5} \mathrm{~cm}^{-3}\right)$. This reflects the stratification seen in electron temperature. The electron density of the denser regions falls off monotonically with approximately the square of the time since nova outburst.

Elemental Abundances. After correction for the ionic abundance of unseen species, we derived the best available elemental abundances for $\mathrm{H}, \mathrm{He}$, $\mathrm{C}, \mathrm{N}, \mathrm{O}, \mathrm{Ne}, \mathrm{Mg}, \mathrm{Al}, \mathrm{Si}, \mathrm{S}$ and $\mathrm{Fe}$ (Table 1). As in other $\mathrm{C} / \mathrm{O}$ novae, there is strong enrichment of $\mathrm{He}$ and $\mathrm{CNO}$, but not of Ne, thereby confirming that it was not a Ne nova. We estimate that the total mass of the ejecta is $\sim 8.4 \times 10^{-5} \mathrm{M}_{o}$ and that most of the CNO originated in the white dwarf rather than the $\mathrm{H}$-rich material accreted from the secondary star.

Secondary Outbursts. During the secondary outburst on Day 150 , the character of the IUE spectrum is very different from the nebular-type spectra before and after. The spectral index changes from -1.9 to -3.5 during the burst, and this is accompanied by the appearance of striking high-velocity P-Cygni features typical of heavy mass loss. There is also evidence of recombination in that CI 1559 and $1658 \mathrm{~A}$ and NI 1493A have returned. The ratio of SiIII] 1892 and CIII] 1909 increases, indicating that these lines come from higher density regions during the outburst. In the optical spectra, the Balmer decrement is very flat, and the ionisation level in the forbidden lines is lower than before. All the [OIII] lines decrease in strength, while [NII] 5755 and 6583 A increase. A Zanstra-like method, based on the HeII/Hbeta ratio, indicated a reduction in the stellar remnant temperature from $100,000 \mathrm{~K}$ to $85,000 \mathrm{~K}$.

We interpret these results in terms of additional material close to the white dwarf, which absorbs most of the ionising photons, allowing recombination to take place in the more distant ejecta. The source of this extra material could either be the result of mass transfer from the secondary star, or a sudden increase in the wind due to a flare in the nuclear burning. The amount of energy which appears in the observable regions implies that the bolometric luminosity has doubled and is super-Eddington during the secondary outburst.

\section{References}

Cameron 1982, "Essays in Nuclear Astrophysics" eds. Barnes et al. CUP p23.

Hassall et al., 1989 (to be submitted to Mon. Not. R. astr. Soc.)

Ögelman et al., 1984, Astrophys. J. Lett. 287, L31.

Stickland et al. 1981, Mon. Not. R. astr. Soc., 197, 107.

Storey et al. 1981, Mon. Not. R. astr. Soc., 185, 27p. 